\title{
Siegel zeros of Eisenstein series
}

\author{
by \\ Joseph Hundley (State College, PA)
}

1. Introduction. The notion of a "Siegel zero" was first introduced in the context of Dirichlet $L$-functions, $L(s, \chi)$, where $\chi$ is a quadratic Dirichlet character of conductor $D$. Roughly speaking, a Siegel zero of $L(s, \chi)$ is a zero on the real axis close to 1 . More precisely, given $\epsilon>0$, an $\epsilon$-Siegel zero of $L(s, \chi)$ is a real number $\beta$ in the interval $\left(1-\epsilon(\log D)^{-1}, 1\right)$ such that $L(\beta, \chi)=0$. The idea of a "Siegel zero" has been generalized to $L$-functions associated with Maass forms by Hoffstein and Lockhart [H-L]. In this context, the conductor $D$ is replaced by $\lambda N+1$, where $\lambda$ and $N$ are the Laplace eigenvalue and level of a Maass form, respectively. See also [R-W], [W].

The study of Siegel zeros of Eisenstein series dates back to at least 1949 when Chowla and Selberg announced [C-S1], among many other results, what is essentially the first half (existence of a Siegel zero) of the main result of this paper in the case of $\mathrm{GL}_{2}$ over $\mathbb{Q}$. We formulate this result in the form that we shall generalize:

Theorem 1 (GL $(2)$ over $\mathbb{Q}$ case). Given $\epsilon>0$, there exists $Y>0$ such that if $y>Y$ then for each $x, E(x+i y, \beta(x, y))=0$ for some $\beta(x, y) \in$ $\left(1-\epsilon(\log y)^{-1}, 1\right)$, and for all $x, \epsilon$, we have

$$
1-\beta(x, y) \sim \frac{3}{\pi y} \quad \text { as } y \rightarrow \infty .
$$

By the time Chowla and Selberg published their proof in [C-S2], a stronger result along the same lines had been proved by Bateman and Grosswald [B-G1], who give what amounts to a fairly precise value of $Y$ such that if $y>Y$ then $E(z, s)$ has a Siegel zero. Recently, Lagarias and Suzuki have given the exact value at which the constant term of $E(z, s)$ develops a Siegel zero. It is very close to Bateman and Grosswald's $Y$. Both [C-S2] and [B-G1] are phrased in terms of the Epstein zeta function of a quadratic form: $E(z, s)$ is $2 y^{s}$ times the Epstein zeta function of the quadratic form

2000 Mathematics Subject Classification: Primary 32N05.

The author was supported by an NSF VIGRE postdoctoral fellowship. 
$(m, n) \mapsto|m z+n|^{2}$. The Epstein zeta function of an $n$-ary quadratic form stands in a similar relationship to an Eisenstein series on $\mathrm{GL}_{n}$ defined using the parabolic subgroup of block upper triangular matrices, and the constant function on the blocks. Siegel zero results for such functions were obtained by Terras [T]. Hoffstein [Ho] considered the case of Hilbert modular Eisenstein series. In this paper, we extend Siegel zero results for Eisenstein series to the generality of Mœglin and Waldspurger [M-W]. However, our result will be weaker than some of the previous authors' in that we will not obtain a precise error term, and in that we will not obtain any estimates for the optimal value of $Y$.

Returning to the case of $E(z, s)$, one of the motivations for the early investigation seems to have been that one may use this theory to link Siegel zeros of Dirichlet $L$-functions, and the class number one problem for imaginary quadratic fields, later resolved by Stark [S2]. Let $k=\mathbb{Q}(\sqrt{-d})$, and let $\zeta_{k}$ denote its Dedekind zeta function. Then

$$
\zeta(s) L\left(s, \chi_{-d}\right)=\zeta_{k}(s)=\frac{1}{\left|\mathfrak{o}_{k}^{\times}\right|} \sum_{C} 2^{s} d^{-s / 2} E\left(z_{C}, s\right),
$$

where the sum is over ideal classes in $k$ and for each $C$, the point $z_{C}$ is the Heegner point associated to $C$. For $d$ large, the point associated to the identity class lies in the region where the Eisenstein series has a Siegel zero. Both of the original pairs of authors noted, in [C-S2, Section 9], and [B-G2] respectively, that if a 10th imaginary quadratic field of class number 1 had existed, the corresponding Dirichlet $L$-function would have had a Siegel zero.

Goldfeld [G] considered formula (1) in general, and showed that the $L\left(s, \chi_{-d}\right)$ has a Siegel zero under a weaker assumption that the class number is small relative to the discriminant, and gave an asymptotic formula for the location of this zero. He also considered the case of real quadratic fields, in which the points $z_{C}$ are replaced by closed geodesics. These asymptotic formulae were the inspiration for the ones in this paper. Additional asymptotic formulae were derived by Goldfeld and Schinzel [G-S].

Concerning the other zeros of $E(z, s)$, Davenport and Heilbronn [D-H] showed that there are infinitely many in the region $\sigma>1$. Here $s=\sigma+i t$. See also [He, p. 89, second remark]. Stark [S1], however, showed that when $y$ is large, all of the zeros in the region $-1 \leq \sigma \leq 2,-2 y \leq t \leq 2 y$ are simple and that, with the exception of the Siegel zero, and a second real zero associated to it by the functional equation, they all lie on the critical line. Hejhal [He, Prop. 5.3(f), p. 86] showed that the constant term of $E(z, s)$ actually satisfies the modified Riemann hypothesis for $y \geq 1$. See the introduction of [L-S] for more on this as well.

The author wishes to thank Carlos Moreno, who suggested that the result, originally proved only for $\mathrm{GL}_{n}$ over $\mathbb{Q}$, would go through in this 
generality, as well as Dorian Goldfeld, Hervé Jacquet, and David Ginzburg, for help and advice along the way. He also wishes to thank the referee for a very helpful report.

1.1. Notation. For the most part, we follow the notation of Møglin and Waldspurger $[\mathrm{M}-\mathrm{W}]$. This entails certain redundancies: for example the symbol $k$ is used both for the global field and an element of the maximal compact, while $M$ is both a Levi subgroup and an intertwining operator. All references are to $[\mathrm{M}-\mathrm{W}]$ until Section 4.

Thus, let $k$ be a global field, $\mathbb{A}$ the adeles of $k, G$ a connected reductive algebraic group defined over $k$, and $\mathbf{G}$ a finite central covering of $G(\mathbb{A})$ such that $G(k)$ lifts to a subgroup of $\mathbf{G}$.

Fix once and for all a choice of minimal parabolic $P_{0}$ of $G$ and a Levi subgroup $M_{0}$ of $P_{0}$, both defined over $k$. This also fixes a definition of "standard" for parabolics and Levis (p. 4). Let $T_{0}$ be the maximal split torus in the center of $M_{0}$ and $\Delta_{0}$ the set of simple positive roots for $T_{0}$ determined by $P_{0}$. Fix a maximal compact subgroup $\mathbf{K}$ of $\mathbf{G}$, as in I.1.4.

Let $P$ be a standard parabolic subgroup of $G$, and let $U$ be its unipotent radical, and $M$ its standard Levi. Then $U(\mathbb{A})$ lifts canonically into $\mathbf{G}$. Let $\mathbf{M}$ denote the preimage of $M(\mathbb{A})$ in $\mathbf{G}$. If $\chi$ is a rational character of $M$, we obtain a map $\mathbf{M} \rightarrow \mathbb{R}_{+}^{\times}$by projecting to $M(\mathbb{A})$, using $\chi$ to get to $\mathbb{A}^{\times}$, and then taking the absolute value. Let $\mathbf{M}^{1}$ denote the intersection of the kernels of all such maps. Let $X_{M}^{\mathbf{G}}$ and $\operatorname{Re} X_{M}^{\mathbf{G}}$ denote the groups of continuous homomorphisms of $\mathbf{M}$ to $\mathbb{C}^{\times}$and $\mathbb{R}_{+}^{\times}$respectively, which are trivial on $\mathbf{M}^{1}$ and on the center of $\mathbf{G}$. When $k$ is a number field, $X_{M}^{\mathbf{G}}$ may be identified with a complex vector space $\left(\mathfrak{a}_{M}^{\mathbf{G}}\right)^{*}$. When $k$ is a function field, it has a finite number of connected components, and there is a natural projection from $\left(\mathfrak{a}_{M}^{\mathbf{G}}\right)^{*}$ to the identity component, which still identifies $\operatorname{Re} X_{M}^{\mathbf{G}}$ with a real vector space. See I.1.4 and I.1.6.

There is unique map $m_{P}: \mathbf{G} \rightarrow \mathbf{M}^{1} \backslash \mathbf{M}$ defined by requiring that if $g=u m k$ for some $u \in U(\mathbb{A}), m \in \mathbf{M}$ and $k \in \mathbf{K}$, then $m_{P}(g)=\mathbf{M}^{1} m$. If $\varphi$ is a function $U(\mathbb{A}) M(k) \backslash \mathbf{G} \mapsto \mathbb{C}$ and $k \in \mathbf{K}$, let us define a function $\varphi_{k}$ on $M(k) \backslash \mathbf{M}$ by $\varphi_{k}(m)=m^{-\varrho_{P}} \varphi(m k)$, where $\varrho_{P}$ is half the sum of the roots of $M$ in Lie $U$. Let $\phi_{\pi}$ be an automorphic form on $U(\mathbb{A}) M(k) \backslash \mathbf{G}$ such that for each $k$, the function $\phi_{\pi, k}$ is a cusp form on $\mathbf{M}$ which generates a semisimple isotypic submodule of type $\pi$, where $\pi$ is an automorphic subrepresentation of $\mathbf{M}$ in the sense of [M-W, p. 78].

For each $\lambda \in X_{M}^{\mathbf{G}}$, let

$$
\lambda \phi_{\pi}(g)=m_{P}(g)^{\lambda} \phi_{\pi}(g)
$$

Then for each $k$, the function $\lambda \phi_{\pi, k}$ is a cusp form on $\mathbf{M}$ which generates a semisimple isotypic submodule of type $\pi \otimes \lambda$. 
For $\lambda$ in a suitable cone in $X_{M}^{\mathbf{G}}$, the Eisenstein series is defined by the following convergent sum:

$$
E\left(\lambda \phi_{\pi}, \pi \otimes \lambda\right)(g)=\sum_{\gamma \in P(k) \backslash G(k)} \lambda \phi_{\pi}(\gamma g) .
$$

It is holomorphic for $\lambda$ in the domain of convergence, and extends to a meromorphic function on all of $X_{M}^{\mathbf{G}}$. We may assume without loss of generality that $\pi$ is unitary. This amounts to, at most, altering our choice of "base point" (see I.3.3). (We deviate from [M-W] in viewing the Eisenstein series as a function on $X_{M}^{\mathbf{G}}$, rather than their $\mathfrak{P}$, which is a principal homogeneous space for the quotient of $X_{M}^{\mathrm{G}}$ by a certain finite group.)

Suppose that $\phi_{\pi}$ is real-valued. Then $E\left(\lambda \phi_{\pi}, \pi \otimes \lambda\right)$ is real-valued for $\lambda \in \operatorname{Re} X_{M}^{\mathbf{G}}$. Let us fix $P$ and $\phi_{\pi}$ once and for all. For each $\alpha \in \Delta_{0}$, let $P_{\alpha}=M_{\alpha} U_{\alpha}$ denote the standard maximal parabolic such that every element of $\Delta_{0}$ is a root of $M_{\alpha}$ except $\alpha$. Our choice of $\Delta_{0}$ determines a set of positive roots for the action of $T_{0}$ on any Levi $M^{\prime}$ which we denote by $R^{+}\left(T_{0}, M^{\prime}\right)$.

Let $W$ denote the Weyl group of $G$, and $W_{M}$ that of $M$. Let $W(M)$ be the set of $w \in W$ of minimal length in their class $w W_{M}$, such that $w M w^{-1}$ is a standard Levi of $G$. Let $W\left(M, M_{\alpha}\right)$ denote the set of $w \in W$ such that $w^{-1} \theta>0$ for every $\theta \in R^{+}\left(T_{0}, M_{\alpha}\right)$ and $w M w^{-1}$ is a standard Levi subgroup of $M_{\alpha}$.

If $k$ is a function field we fix once and for all a place $v_{0}$, and a uniformizing parameter $\mathfrak{w}$, and let $q=|\mathfrak{w}|_{v_{0}}^{-1}$. Let $\mathfrak{m}=\mathbb{R}_{+}^{\times}$in the number field case, or $\mathfrak{w}^{\mathbb{Z}}$ in the function field case. Thus $\mathfrak{m}$ may be embedded in $\mathbb{A}^{\times}$either at $k_{v_{0}}$ or diagonally at the infinite places, as a subgroup on which the absolute value is injective. One then has a subgroup of $T_{0}(\mathbb{A})$ isomorphic to $\mathfrak{m}^{R}$, where $R$ is the rank of $T_{0}$, and in I.2.1 of [M-W] this is extended to a subgroup $A_{\mathbf{M}_{0}}$ of $\mathbf{G}$, still isomorphic to $\mathfrak{m}^{R}$. We then define

$$
A_{\mathbf{M}_{\alpha}}=A_{\mathbf{M}_{0}} \cap Z_{\mathbf{M}_{\alpha}}
$$

If $\lambda \in \operatorname{Re} X_{M}^{\mathbf{G}}$, then $\left.\lambda\right|_{A_{\mathbf{M}_{\alpha}}}$ factors through the quotient $A_{\mathbf{M}_{\alpha}} / A_{\mathbf{G}}$, and is trivial on any torsion in this quotient. It follows that we may fix a map $\widetilde{\alpha}: \mathfrak{m} \rightarrow A_{\mathbf{M}_{\alpha}}$ such that $\left.\lambda\right|_{A_{\mathbf{M}_{\alpha}}}$ is determined by $\lambda \circ \widetilde{\alpha}$. In the function field case, we also denote by $\widetilde{\alpha}$ the composition of $\widetilde{\alpha}$ with $|\cdot| v_{v_{0}}^{-1}: q^{\mathbb{Z}} \rightarrow \mathfrak{w}^{\mathbb{Z}}$. Then $\lambda \circ \widetilde{\alpha}$ is a continuous homomorphism of a subgroup of $\mathbb{R}_{+}^{\times}$to $\mathbb{R}_{+}^{\times}$, so we may define $\langle\widetilde{\alpha}, \lambda\rangle$ to be the unique real number such that

$$
\lambda \circ \widetilde{\alpha}(y)=y^{\langle\widetilde{\alpha}, \lambda\rangle} .
$$

Replacing $\widetilde{\alpha}$ by the map $y \mapsto \widetilde{\alpha}\left(y^{-1}\right)$ if necessary, we may add the stipulation that $\langle\widetilde{\alpha}, \alpha\rangle>0$. We will also need to refer to the Siegel set $S$, defined in Section I.2.1 of [M-W], using a compact subset $\omega$ of $\mathbf{P}_{0}$. 
Definition. Let us say that a map $\Lambda: \mathbb{C} \rightarrow X_{M}^{\mathbf{G}}$ is elementary if it is holomorphic and the restriction to $\mathbb{R}$ is an affine map into the real vector space $\operatorname{Re} X_{M}^{\mathbf{G}}$.

The constant term of $E\left(\lambda \phi_{\pi}, \lambda \otimes \pi\right)$ along $P_{\alpha}$ (see I.2.6) is given in terms of Eisenstein series $E^{M_{\alpha}}$, defined analogously, with $M_{\alpha}$ replacing $G$, and intertwining operators $M(w, \pi)$ defined in II.1.6:

$$
E_{P_{\alpha}}\left(\lambda \phi_{\pi}, \lambda \otimes \pi\right)=\sum_{w \in W\left(M, M_{\alpha}\right)} E^{M_{\alpha}}\left(M(w, \lambda \otimes \pi) \lambda \phi_{\pi}, w(\lambda \otimes \pi)\right)
$$

(see II.1.7).

Suppose that $E$ has a singularity along a root hyperplane $H$, associated to a root $\theta$ as in IV.1.6. Our theorem applies only to the case when $H_{\mathbb{R}}:=$ $H \cap \operatorname{Re} X_{M}^{\mathbf{G}}$ is nonempty. In this case, $H_{\mathbb{R}}$ will be of the form

$$
\left\{\lambda \in \operatorname{Re} X_{M}^{\mathbf{G}}:\langle\lambda, \check{\theta}\rangle=c_{H}\right\},
$$

for some $c_{H} \in \mathbb{R}$. See IV.1.6 and I.1.11. Here $\check{\theta}$ may be taken to be the coroot associated to a positive root $\theta$, or its projection to the dual of $\operatorname{Re} X_{M}^{\mathrm{G}}$ : the set $H$ is the same either way. We say that $\lambda \in H$ is generic for $\alpha$ if $\lambda$ does not lie in any other hyperplane along which $E$ has a singularity, and $\left\langle\widetilde{\alpha}, w_{1} \lambda\right\rangle=\left\langle\widetilde{\alpha}, w_{2} \lambda\right\rangle$ for $w_{1}, w_{2} \in W\left(M, M_{\alpha}\right)$ iff $w_{1}=w_{2}$. It follows that for $W\left(M, M_{\alpha}\right)$ nonempty, and $\lambda$ generic for $\alpha$, there is a unique $w_{\max }(\lambda, \alpha)$ such that $\langle\widetilde{\alpha}, w \lambda\rangle$ is maximal.

\subsection{Statement of main result}

THEOREM 2. Fix a root hyperplane $H$ along which the Eisenstein series is singular, a root $\alpha$, an elementary map $\Lambda$ such that $\Lambda(\mathbb{C}) \cap H=\Lambda(0)$ is generic for $\alpha$, and elements $p \in \omega, k \in \mathbf{K}$. Let

$$
E(y, s)=E\left(\Lambda(s) \phi_{\pi}, \Lambda(s) \otimes \pi\right)(p \widetilde{\alpha}(y) k),
$$

and for each $w \in W\left(M, M_{\alpha}\right)$, let

$$
E_{w}(y, s)=E^{M_{\alpha}}\left(M(w, \Lambda(s) \otimes \pi) \Lambda(s) \phi_{\pi}, w(\Lambda(s) \otimes \pi)\right)(p \widetilde{\alpha}(y) k) .
$$

We say that $\beta$ is an $\epsilon$-Siegel zero of $E(y, \sigma)$ if $\beta \in\left(-\epsilon(\log y)^{-1}, \epsilon(\log y)^{-1}\right)$ and $E(y, \beta)=0$. Let $W_{\mathrm{sng}}(H, \alpha, \Lambda, p k)=\left\{w \in W(M, M \alpha): E_{w}(1, s)\right.$ has a pole at $s=0\}$. Suppose that $H, \alpha, p, k$, and $\Lambda$ satisfy:

1. $W\left(M, M_{\alpha}\right) \neq \emptyset$ and $w_{\max }(\Lambda(0), \alpha) \notin W_{\mathrm{sng}}(H, \alpha, \Lambda, p k)$.

2. $E_{w_{\max }(\Lambda(0), \alpha)}(1,0) \neq 0$.

3. $E(y, s)$ has a simple pole at $s=0$.

Then we have the following:

(A) If $W_{\mathrm{sng}}(H, \alpha, \Lambda, p k) \neq \emptyset$, let $w_{\mathrm{ms}}$ be the unique element of $W_{\mathrm{sng}}$ such that $\left\langle\widetilde{\alpha}, w_{\mathrm{ms}} \Lambda(0)\right\rangle$ is maximal (among elements of $W_{\mathrm{sng}}$ ), and denote $w_{\max }(\Lambda(0), \alpha)$ more briefly by $w_{\max }$. Then: 
(i) There exists $Y>0$ (dependent on $\epsilon, H, \alpha, p, k, \Lambda$, and $\phi_{\pi}$ ), such that for all $y>Y, E(y, \sigma)$ has an $\epsilon$-Siegel zero.

(ii) Let $\beta:(Y, \infty) \rightarrow \mathbb{R}$, or $\beta: q^{\mathbb{Z}} \cap(Y, \infty) \rightarrow \mathbb{R}$ in the function field case, be any function such that for each $y, \beta(y)$ is an $\epsilon$-Siegel zero of $E(y, \sigma)$. Then $\beta(y) \sim e y^{-\left\langle\widetilde{\alpha}, w_{\max } \Lambda(0)-w_{\operatorname{ms}} \Lambda(0)\right\rangle}$, where

$$
\begin{aligned}
e= & \frac{\chi_{\pi}\left(w_{\mathrm{ms}}^{-1} \widetilde{\alpha}(y) w_{\mathrm{ms}}\right)}{\chi_{\pi}\left(w_{\max }^{-1} \widetilde{\alpha}(y) w_{\max }\right)} \times \\
& \frac{\left(\operatorname{Res}_{s=0} E^{M_{\alpha}}\left(M\left(w_{\mathrm{ms}}, \Lambda(s) \otimes \pi\right) \Lambda(s) \phi_{\pi}, w_{\mathrm{ms}}(\Lambda(s) \otimes \pi)\right)(p k)\right)}{E^{M_{\alpha}}\left(M\left(w_{\max }, \Lambda(0) \otimes \pi\right) \Lambda(0) \phi_{\pi}, w_{\max }(\Lambda(0) \otimes \pi)\right)(p k)} .
\end{aligned}
$$

(B) If $W_{\mathrm{sng}}(H, \alpha, \Lambda, p k)=\emptyset$, then the conclusion is weaker: if $y>Y$ then either $E(y, \sigma)$ has an $\epsilon$-Siegel zero, or $E(y, \sigma)$ is holomorphic at zero. If $k$ is a function field, then it is always the latter.

We will also prove the following lemma, relevant to the problem of choosing $\alpha, \Lambda, p$ and $k$ so that conditions $1-3$ above are satisfied.

Lemma 3. For $\alpha \in \Delta_{0}$ such that $\alpha \notin R^{+}\left(T_{0}, M\right)$ and $\theta \notin R^{+}\left(T_{0}, M_{\alpha}\right)$, we have:

1. The identity element, 1 , is in $W\left(M, M_{\alpha}\right)$.

2. For all $p, k$ and $\Lambda, 1 \notin W_{\text {sng }}(H, \alpha, \Lambda, p k)$.

3. If $c_{H}>0$, then $\left\{\lambda \in H_{\mathbb{R}}\right.$ generic for $\left.\alpha: w_{\max }(\lambda, \alpha)=1\right\}$ is a nonempty open subset of $H_{\mathbb{R}}$.

It is also known (IV.1.11(c)) that when $c_{H}>0$, the singularity along $H$ is without multiplicity. For all $p, k$ the restriction of $E_{1}$ and the continuation of $h_{H}(\lambda) E$ are meromorphic functions of $\lambda \in H$. As we see in an example below, either or both may be trivial (i.e., zero for all $\lambda$ ) for certain values of $p, k$. But, for any $p, k$ such that both are nontrivial, $\Lambda$ may then be chosen so that all three conditions are satisfied.

2. Basic analytic result. Our approach to proving the existence of Siegel zeros of Eisenstein series is as follows. First, we show that any function that can be written in a certain form will have a zero on the real axis very close to its pole. Then, we show that our Eisenstein series can always be put into that form. The lengthy definition of the function $F(y, s)$ in the following lemma should, therefore, be taken as the "mold" into which we will fit the Eisenstein series. As we see here, any function which fits into this mold will have Siegel zeros.

LEMMA 4. Fix real numbers $a, b, c$, and $d$ such that $b>d$. Let $\sigma$ be a real variable, which we may think of as restricted to a small neighborhood of 0 , and let $y$ be another real variable, which we think of as positive and large, keeping in mind that when we apply this lemma to the function field case, 
$y$ will only range over $q^{\mathbb{Z}}$. Let $A(\sigma)$ and $C(\sigma)$ be two real-valued functions that are both continuous and nonvanishing for $\sigma$ in a neighborhood of 0 , and let $B(y, \sigma)$ and $D(y, \sigma)$ be two more real-valued functions, which are both continuous in $\sigma$, and such that $B(y, \sigma) y^{-(a \sigma+b)}$ and $D(y, \sigma) y^{-(c \sigma+d)}$ tend to zero as y tends to $\infty$ for all values of $\sigma$ in some neighborhood of 0 , and that convergence is uniform as $\sigma$ ranges over this neighborhood.

Define:

$$
F(y, \sigma)=\left(A(\sigma) y^{a \sigma+b}+B(y, \sigma)\right)+\frac{1}{\sigma}\left(y^{c \sigma+d} C(\sigma)+D(y, \sigma)\right) .
$$

Then we have:

(i) For every $\epsilon>0$, there exists $Y(\epsilon)>0$ such that if $y>Y(\epsilon)$, then $F(y, \sigma)$ has a zero in the interval $\left(-\epsilon(\log y)^{-1}, \epsilon(\log y)^{-1}\right)$.

(ii) Now fix an $\epsilon>0$, and take $\beta:(Y(\epsilon), \infty) \rightarrow \mathbb{R}$ such that for each $y$ we have $\beta(y) \in\left(-\epsilon(\log y)^{-1}, \epsilon(\log y)^{-1}\right)$, and $F(\beta(y), y)=0$. For any such $\beta$,

$$
-\beta(y) \sim \frac{C(0)}{y^{b-d} A(0)} \quad \text { as } y \rightarrow \infty .
$$

Proof. By replacing $F(y, \sigma)$ with $y^{-(c \sigma+d)} F(y, \sigma)$, which has the same zeros, we may assume $c=d=0$. By considering the four functions $\pm F(y, \pm \sigma)$ we may assume that $A(0)$ and $C(0)$ are positive.

Next, choose $\delta, Y_{1}, m, M$ such that

$$
y>Y_{1},|\sigma|<\delta \Rightarrow\left\{\begin{array}{l}
0<m<A(\sigma)<M \\
0<m<C(\sigma)<M \\
|D(y, \sigma)|<m / 2 \\
|B(y, \sigma)|<(m / 2) y^{a \sigma+b}
\end{array}\right.
$$

Then, for each $y>Y_{1}$, the function $A(\sigma) y^{a \sigma+b}+B(y, \sigma)$ is bounded on $|\sigma|<\delta$, while $C(\sigma)+D(y, \sigma)$ is bounded away from zero. Hence, for every such $y$, there is a neighborhood of the form $(-l, 0)$ on which $F(y, \sigma)<0$. Now suppose that $y>Y_{1}$, and $\epsilon(\log y)^{-1}<\delta$. Then the bounds $(3)$ are valid at $\sigma=-\epsilon(\log y)^{-1}$, yielding

$$
F\left(y,-\epsilon(\log y)^{-1}\right)>\frac{m}{2} e^{-a \epsilon} y^{b}-\epsilon^{-1}\left(M+\frac{m}{2}\right) \log y .
$$

Clearly, if we fix an $\epsilon>0$, we may first choose $Y_{2} \geq Y_{1}$ such that the above is valid for all $y>Y_{2}$, and then choose $Y_{3}$ such that if $y>Y_{3}$, the right side is positive. We then let $Y=\max \left(Y_{2}, Y_{3}\right)$, and the first assertion is proved. Moreover, if we fix an $\epsilon^{\prime}<\epsilon$, we can choose $Y_{2}^{\prime}$ such that for every $y>Y_{2}^{\prime}, \epsilon^{\prime}<\varepsilon<\epsilon$, we have $\varepsilon(\log y)^{-1}<\delta$, and then choose $Y_{3}^{\prime}$ such that 
for $y>Y_{3}^{\prime}, \epsilon^{\prime}<\varepsilon<\epsilon$, we have

$$
\frac{m}{2} e^{-a \varepsilon} y^{b}-\varepsilon^{-1}\left(M+\frac{m}{2}\right) \log y>0 .
$$

It follows that any $\beta(y)$, defined as above with respect to $\epsilon$, satisfies

$$
\beta(y) \in\left(-\epsilon^{\prime}(\log y)^{-1}, 0\right) \quad \text { for } y>Y^{\prime} .
$$

Since this works for any $\epsilon^{\prime}$, we have

$$
\lim _{y \rightarrow \infty} y^{-\beta(y)}=1
$$

independently of the choice of $\epsilon$, and of any possible choice of $\beta(y)$.

To prove (ii), we note that $F(y, \beta(y))=0$ iff

$$
A(\beta(y)) y^{a \beta(y)+b}+B(y, \beta(y))=\frac{-1}{\beta(y)}(C(\beta(y))+D(y, \beta(y))) .
$$

We have seen that for $\beta(y)$ near 0 and $y$ large the left side is nonzero, so we may put this into the form

$$
\beta(y)=-\frac{C(\beta(y))+D(y, \beta(y))}{A(\beta(y)) y^{a \beta(y)+b}+B(y, \beta(y))} .
$$

So

$$
\begin{aligned}
\lim _{y \rightarrow \infty} & \frac{C(0) /\left(A(0) y^{b}\right)}{-\beta(y)} \\
& =\lim _{y \rightarrow \infty} \frac{C(0)}{C(\beta(y))+D(y, \beta(y))} \frac{\left(A(\beta(y)) y^{a \beta(y)+b}+B(y, \beta(y))\right) y^{-b}}{A(0)} .
\end{aligned}
$$

The second limit is evidently 1 , which proves the asymptotic formula.

\section{REMARKS.}

1. If, on the other hand, $F$ is in the same form with $b<d$, but all the other assumptions are the same, then similar arguments show that $F(y, \sigma)$ will be nonvanishing for $\sigma$ in a neighborhood of 0 and $y$ sufficiently large.

2. If $F$ is as above, but $C(0)=0$, then the asymptotic formula is no longer correct, but the Siegel zero still exists for all values of $y>Y$ such that $D(y, \sigma) \neq 0$.

2.1. Necessary fact. We will need one more well known fact from the theory of Eisenstein series: the Eisenstein series is well approximated by its constant term.

Proposition 5. If $k$ is a function field, then there is a constant $c$ such that $E\left(\lambda \phi_{\pi}, \pi \otimes \lambda\right)(g)-E_{P_{\alpha}}\left(\lambda \phi_{\pi}, \pi \otimes \lambda\right)(g)=0$, whenever $g \in S$ and $m_{P_{0}}(g)^{\alpha}>c$, where $S$ is a Siegel domain as in I.2.1. In particular, for 
$\Lambda, p, k, \widetilde{\alpha}$ as in the main theorem,

$$
\left(E-E_{P_{\alpha}}\right)\left(\Lambda(\sigma) \phi_{\pi}, \pi \otimes \Lambda(\sigma)\right)(p \widetilde{\alpha}(y) k)=0,
$$

for $p \in \omega, k \in K$ and $y$ sufficiently large. If $k$ is a number field, then for $\Lambda$, $p, k, \widetilde{\alpha}$ as in the main theorem,

$$
\sigma\left(\left(E-E_{P_{\alpha}}\right)\left(\Lambda(\sigma) \phi_{\pi}, \Lambda(\sigma) \otimes \pi\right)(p \widetilde{\alpha}(y) k)\right)
$$

is rapidly decreasing as a function of y for $p \in \mathbf{G}^{1} \cap \omega, k \in K$, uniformly for $\sigma$ in a neighborhood of zero.

Proof. The function field case is immediate from Lemma I.2.7. For the number field case we use Lemma I.2.10. Lemma I.4.4, in conjunction with I.2.5, yields the bounds required by the hypotheses of Lemma I.2.10.

\section{Proofs}

3.1. Proof of Theorem 2. As $H, \alpha, \Lambda, p$ and $k$ are fixed, we suppress them from the notation, denoting $W_{\text {sng }}(H, \alpha, \Lambda, p k)$ by $W_{\text {sng }}$, etc. The idea is to fit $E(y, \sigma)$ into the mold described in Section 2. In the new notation we have introduced, equation (2) reads

$$
E_{P_{\alpha}}(y, \sigma)=\sum_{w \in W\left(M, M_{\alpha}\right)} E_{w}(y, \sigma) .
$$

We observe that

$$
E_{w}(y, \sigma)=\chi_{\pi}\left(w^{-1} \widetilde{\alpha}(y) w\right) y^{\left\langle\widetilde{\alpha}, \varrho_{P_{\alpha}}+w \Lambda(\sigma)\right\rangle} E_{w}(1, \sigma) .
$$

Now, $\chi_{\pi}$ is both real-valued and unitary. When $k$ is a number field, the function $\chi_{\pi}\left(w^{-1} \widetilde{\alpha}(y) w\right)$ is trivial, but when $k$ is a function field, $\chi_{\pi}\left(w^{-1} \widetilde{\alpha}\left(q^{n}\right) w\right)$ may equal -1 for some $w$ when $n$ is odd. In this case, one may consider restrictions to odd and even $n$ separately, and combine the results. We omit the details, and assume that $\chi_{\pi}\left(w^{-1} \widetilde{\alpha}(y) w\right)$ is identically 1 when $w$ is either $w_{\max }$ or $w_{\text {ms }}$.

For each $w$, the map $\sigma \mapsto\langle\widetilde{\alpha}, w \Lambda(\sigma)\rangle$ is an affine map $\mathbb{R} \rightarrow \mathbb{R}$, and so we may define $a, b, c$, and $d$ by the conditions $\left\langle\widetilde{\alpha}, \varrho_{P_{\alpha}}+w_{\max } \Lambda(\sigma)\right\rangle=a \sigma+b$, and, if $W_{\text {sng }}$ is nonempty, $\left\langle\widetilde{\alpha}, \varrho_{P_{\alpha}}+w_{\text {ms }} \Lambda(\sigma)\right\rangle=c \sigma+d$. If $W_{\text {sng }}$ is empty, we take $c=0$, and $d$ any real number less than $b$. We also take

$$
\begin{aligned}
A(\sigma) & =E_{w_{\max }}(1, \sigma), \\
C(\sigma) & =\sigma E_{w_{\mathrm{ms}}}(1, \sigma), \text { or } 0 \text { if } W_{\mathrm{sng}}=\emptyset, \\
D(y, \sigma) & =\sigma\left(\sum_{w \in W_{\mathrm{sng}}-\left\{w_{\mathrm{ms}}\right\}} E_{w}(y, \sigma)+\left(E-E_{P_{\alpha}}\right)(y, \sigma)\right), \\
B(y, \sigma) & \sum_{w \in W\left(M, M_{\alpha}\right)-W_{\mathrm{sng}}-\left\{w_{\max }\right\}} E_{w}(y, \sigma),
\end{aligned}
$$

where we extend $C$ and $D$ to $\sigma=0$ by continuity. 
We check that all the hypotheses of Lemma 4 are satisfied. As $\Lambda(0)$ is generic, we may choose an open ball containing it which intersects no other hyperplane along which $E$ is singular. The continuity of each function on the set of $\sigma$ mapping into this ball is clear. The fact that all functions are real-valued follows from the fact that $\phi_{\pi}$ is. Condition 2 of Theorem 2 is precisely that $A(0) \neq 0$, and in the case when $W_{\text {sng }} \neq \emptyset$, the value of $C$ at 0 is the residue of $E_{w_{\mathrm{ms}}}(1, \sigma)$ at zero. The bounds on $B$ and $D$ as $y \rightarrow \infty$ follow easily from (5) and Proposition 5.

3.2. Proof of Lemma 3. The first assertion is trivial. The second and third follow from more general assertions, which we now prove. For $w \in$ $W\left(M, M_{\alpha}\right)$, let $W_{M_{\alpha}}\left(w M w^{-1}\right)$ be defined in the same way as $W(M)$, with $M_{\alpha}$ replacing $G$ and $w M w^{-1}$ replacing $M$.

Lemma 6. Let $H$ be a root hyperplane associated to a root $\theta$, and fix $\alpha$. If there exist $p, k, \Lambda$ such that $\Lambda(0) \in H$ is generic for $\alpha$, and $w \in$ $W_{\text {sng }}(H, \alpha, p, k, \Lambda)$, then there exists $w^{\prime} \in W_{M_{\alpha}}\left(w M w^{-1}\right)$ such that $w^{\prime} w \theta<0$.

Proof. For some $w^{\prime} \in W_{M_{\alpha}}\left(w M w^{-1}\right)$, write $w^{\prime} w=s_{\gamma_{l}} \cdots s_{\gamma_{1}}$, where for each $i, s_{\gamma_{i}}$ is an "elementary symmetry" as in Section I.1.8. See also Section IV.4.1. Let $w(j)=s_{\gamma_{j}} \cdots s_{\gamma_{1}}$. Then

$$
M\left(w^{\prime} w, \pi \otimes \lambda\right)=M\left(s_{\gamma_{l}}, w(l-1)(\pi \otimes \lambda)\right) \circ \cdots \circ M\left(s_{\gamma_{1}}, \pi \otimes \lambda\right) .
$$

The singularities of $M\left(s_{\gamma}, \tau \otimes \mu\right)$ are carried by a locally finite set of hyperplanes associated to $\gamma$. Hence the singularities of $M\left(s_{\gamma_{i}}, w(i-1)(\pi \otimes \lambda)\right)$ are carried by a locally finite set of root hyperplanes associated to the root $w(i-1)^{-1} \gamma_{i}$, and, in general, the singularities of $M\left(w^{\prime} w, \pi \otimes \lambda\right)$ are carried by a locally finite set of root hyperplanes each of which is associated to a root $\gamma$ such that $w^{\prime} w \gamma<0$.

Suppose that $w^{\prime} w \theta>0$ for all $w^{\prime} \in W_{M_{\alpha}}\left(w M w^{-1}\right)$. Then there is a locally finite set of root hyperplanes, not containing $H$, that carries the singularities of $M\left(w w^{\prime}, \pi \otimes \lambda\right)$ for every $w^{\prime}$. Hence it carries the singularities of all the cuspidal components of all the constant terms of $E^{M_{\alpha}}(M(w$, $\pi \otimes \lambda) \lambda \phi_{\pi}, w(\pi \otimes \lambda)$ ) (equation (2) above, and IV.1.9(b)). By I.4.10 it carries the singularities of $E^{M_{\alpha}}\left(M(w, \pi \otimes \lambda) \lambda \phi_{\pi}, w(\pi \otimes \lambda)\right)$ as well. The result follows.

Under the hypotheses of Lemma 3, $w^{\prime} \theta>0$ for all $w^{\prime} \in W_{M_{\alpha}}(M)$, and 2 follows. As for 3 , it is clear that the set in question is always open, and that it is empty iff $\left\{\lambda \in H_{\mathbb{R}}: w_{\max }(\lambda, \alpha)=1\right\}$ is. Thus, we only need to prove that this latter set is nonempty.

Lemma 7. If $c_{H}>0$, and $1 \in W\left(M, M_{\alpha}\right)$, then there exists $\lambda \in H_{\mathbb{R}}$ such that $w_{\max }(\lambda, \alpha)=1$. 
Proof. The linear functional on $\Delta_{0}$ given by pairing with $\widetilde{\alpha}$ corresponds to a positive multiple of the one given by pairing with the fundamental coweight $\widehat{\varpi}_{\alpha}$, in the basis dual to $\Delta_{0}$. Given an element $\lambda$ of $\operatorname{Re} X_{M_{0}}^{\mathrm{G}}$ which is irrational, i.e., has trivial kernel in the coweight lattice, we can define notions of $\lambda$-positivity for coroots and $\lambda$-dominance for coweights. If the definitions of positivity for coroots that come from $\lambda$ and $\Delta_{0}$ coincide, then the definitions of dominance for coweights will as well. Hence, whenever

$$
\langle\lambda, \check{\gamma}\rangle>0 \quad \forall \gamma \in \Delta_{0}
$$

and $\lambda$ is irrational, we have

$$
\langle\lambda, \widetilde{\alpha}\rangle \geq\langle w \lambda, \widetilde{\alpha}\rangle \quad \forall w \in W,
$$

with equality only if $w^{-1} \widetilde{\alpha}=\widetilde{\alpha}$. Now project $\lambda$ to $\bar{\lambda} \in \operatorname{Re} X_{M}^{\mathbf{G}}$. This projection corresponds to restriction from $Z_{\mathbf{M}_{0}}$ to $Z_{\mathbf{M}}$ (see pp. 7,11). Since the image of $w^{-1} \widetilde{\alpha}$ is contained in $Z_{\mathbf{M}}$ whenever $w \in W\left(M, M_{\alpha}\right)$, we find that

$$
\langle\bar{\lambda}, \widetilde{\alpha}\rangle \geq\langle w \bar{\lambda}, \widetilde{\alpha}\rangle \quad \forall w \in W\left(M, M_{\alpha}\right),
$$

with equality only if $w=1$. Thus, for $\bar{\lambda}$ the projection of any $\lambda$ as above, we have $w_{\max }(\bar{\lambda}, \alpha)=1$. Now, let $\gamma_{\theta}$ be the root associated to $\theta$ as in Section I.1.11 (so that $\check{\theta}$ is defined as the projection of $\check{\gamma_{\theta}}$ to $\operatorname{Re} \mathfrak{a}_{M}$ ). It should be emphasized that $\left\langle\lambda, \check{\gamma}_{\theta}\right\rangle$ and $\langle\bar{\lambda}, \check{\theta}\rangle$ need not be equal. However, $\langle\lambda, \check{\theta}\rangle$ and $\langle\bar{\lambda}, \check{\theta}\rangle$ are always equal. So, it is enough to verify that for some irrational $\lambda$, with $\langle\lambda, \check{\gamma}\rangle>0 \forall \gamma \in \Delta_{0}$, the quantity $\langle\lambda, \check{\theta}\rangle$ is also positive. This is straightforward, when $\check{\theta}$ is written in terms of the basis $\Delta_{0}^{\vee}$ of coroots $\check{\gamma}$, and $\lambda$ in terms of the dual basis.

4. Examples. Let us consider the example when $G=\mathrm{GL}(n)$, the representation $\pi$ is unramified, and we choose $\phi_{\pi}$ to be the spherical vector. In this case, the intertwining operators may be given explicitly in terms of automorphic $L$-functions, as in [L]. It follows that a pole of the Eisenstein series comes from either a zero or a pole of one of these $L$-functions. The zeros are quite mysterious, but the poles of Rankin-Selberg $L$-functions on GL( $n)$ have been completely determined by Jacquet and Shalika [J-S1], [J-S2], and referring back to the $\mathrm{GL}(2)$ case, we see that it is these poles that provide the correct analog of the pole of the GL(2) Eisenstein series at 1.

As noted, the case $G=\mathrm{GL}(2)$ is the simplest example of our theorem. Let us consider two more which exhibit some of the features of the general case, but are not so complicated as to become unwieldy.

4.1. Example one: $\mathrm{GL}(4)$ over $\mathbb{Q}$. First let us consider the case $k=\mathbb{Q}$ and $\mathbf{G}=\mathrm{GL}(4, \mathbb{A})$. We take the usual Borel subgroup, consisting of all upper triangular elements, and let $P$ be the standard maximal parabolic such that $M \cong \mathrm{GL}(2) \times \mathrm{GL}(2)$. 
In this case, $\operatorname{Re} X_{M}^{G}$ is one-dimensional, consisting of all real powers of the modulus

$$
\left(\begin{array}{cc}
h_{1} & * \\
& h_{2}
\end{array}\right) \mapsto\left|\frac{\operatorname{det} h_{1}}{\operatorname{det} h_{2}}\right|^{2},
$$

so we identify it with $\mathbb{R}$. In order to have good knowledge of the poles of the intertwining operators, we will want to make a suitable choice of $\pi$ and $\phi_{\pi}$, which can also be described quite explicitly.

Let $\varphi$ be a real-valued Maass Hecke eigenform of level 1, right invariant by the center and the maximal compact, and define a function on $\operatorname{GL}(4, \mathbb{A})$ by

$$
I\left(\left(\begin{array}{cc}
h_{1} & * \\
& h_{2}
\end{array}\right) k, s, \varphi\right)=\left|\frac{\operatorname{det} h_{1}}{\operatorname{det} h_{2}}\right|^{2 s+1} \varphi\left(h_{1}\right) \varphi\left(h_{2}\right)
$$

for $k$ now in the maximal compact of $\mathrm{GL}(4, \mathbb{A})$. Our Eisenstein series is

$$
E(g, s, \varphi)=\sum_{\gamma \in P(\mathbb{Q}) \backslash \mathrm{GL}(4, \mathbb{Q})} I(\gamma g, s, \varphi) .
$$

In this case $W\left(M, M^{\prime}\right)=\emptyset$, unless $M^{\prime}=M$, and

$$
E_{P}(g, s, \varphi)=I(g, s, \varphi)+\frac{L(2 s, \varphi \times \varphi)}{L(2 s+1, \varphi \times \varphi)} I(g,-s, \varphi) .
$$

Here $L$ denotes the completed $L$-function-including gamma factors. (This is essentially a special case of the computation in [L].) Since $\operatorname{Re} X_{M}^{G}$ is onedimensional, a codimension 1 subspace is a point. Thus our "root hyperplane" is just $s=1 / 2$, or some zero of $L(2 s+1, \varphi \times \varphi)$. We consider the one at $1 / 2$, and let $\Lambda(\sigma)=\sigma+1 / 2$, and $\widetilde{\alpha}(y)=\operatorname{diag}(y, y, 1,1)$, embedded at the infinite place. Note that if $p=\left(\begin{array}{cc}h_{1} & * \\ & h_{2}\end{array}\right)$ with $\varphi\left(h_{1}\right)$ or $\varphi\left(h_{2}\right)=0$, then the constant term is zero for all $s$. For any other $p, W_{\text {sng }}=\left\{\left({ }_{I}{ }^{I}\right)\right\}$. For $\sigma$ positive, $w_{\max }=I$, and the theorem applies.

Let

$$
\begin{aligned}
A(\sigma) & =I(p k, \sigma+1 / 2, \varphi), \\
C(\sigma) & =\frac{L(2 \sigma+1, \varphi \times \varphi)}{L(2 \sigma+2, \varphi \times \varphi)} I(p k,-\sigma-1 / 2, \varphi), \\
D(y, \sigma) & =E(p \widetilde{\alpha}(y) k, \sigma+1 / 2, \varphi)-E_{P}(p \widetilde{\alpha}(y) k, \sigma+1 / 2, \varphi) .
\end{aligned}
$$

Let $a=4, b=4, c=-4$ and $d=0$. The main analytic lemma applies. The asymptotic formula it produces is

$$
-\beta \sim \frac{(\varphi, \varphi)}{2 L(2, \varphi \times \varphi)}\left|\frac{\operatorname{det} h_{1}}{\operatorname{det} h_{2}}\right|^{-2} y^{-4},
$$

where $(\varphi, \varphi)$ is the Petersson inner product of $\varphi$ with itself. 
4.2. Example two: GL(3) over $\mathbb{Q}$. Now let us consider the case $k=\mathbb{Q}$ and $\mathbf{G}=\mathrm{GL}(3, \mathbb{A})$. We take $P=P_{0}$ to be the Borel subgroup of upper triangular matrices and $M=T_{0}$ to be the torus of diagonal matrices. The associated set of simple positive roots is $\{\alpha, \theta\}$ defined by

$$
\alpha\left(\left(\begin{array}{ccc}
t_{1} & & \\
& t_{2} & \\
& & t_{3}
\end{array}\right)\right)=\frac{t_{1}}{t_{2}}, \quad \theta\left(\left(\begin{array}{ccc}
t_{1} & & \\
& t_{2} & \\
& & t_{3}
\end{array}\right)\right)=\frac{t_{2}}{t_{3}} .
$$

We take $\pi$ to be the trivial representation, and $\phi_{\pi}$ to be the constant function 1 . We parametrize $\operatorname{Re} X_{M}^{\mathbf{G}}$ by associating the map

$$
\mathcal{I}_{\lambda}\left[\left(\begin{array}{ccc}
t_{1} & & \\
& t_{2} & \\
& & t_{3}
\end{array}\right)\right]=\left|t_{1}\right|^{\lambda_{1}+1}\left|t_{2}\right|^{\lambda_{2}}\left|t_{3}\right|^{\lambda_{3}-1}
$$

to the triple $\lambda=\left(\lambda_{1}, \lambda_{2}, \lambda_{3}\right) \in \mathbb{R}^{3}$ such that $\lambda_{1}+\lambda_{2}+\lambda_{3}=0$.

We work with $\alpha$, and define the map $\widetilde{\alpha}$ by

$$
\widetilde{\alpha}(y)=\left(\begin{array}{ccc}
y & & \\
& 1 & \\
& & 1
\end{array}\right),
$$

embedded at the infinite place. The Weyl group $W$ of $G$ may be identified with the group of permutation matrices. Then

$$
W\left(M, M_{\alpha}\right)=\left\{I,\left(\begin{array}{lll} 
& 1 & \\
1 & & \\
& & 1
\end{array}\right)\left(\begin{array}{lll} 
& & 1 \\
1 & & \\
& 1
\end{array}\right)\right\} .
$$

We compute the intertwining operators as usual, by reducing from the general case to the relative rank one case, i.e., to a Levi isomorphic to GL(2). As on GL(2), each root contributes a ratio of Riemann zeta functions. Specifically, if $\zeta^{*}(s)=\pi^{-s / 2} \Gamma(s / 2) \zeta(s)$ is the "completed" Riemann zeta function, then we have

$$
\begin{aligned}
& M\left(\left(\begin{array}{lll}
1 & & \\
& & 1
\end{array}\right), \mathcal{I}_{\lambda}\right) \mathcal{I}_{\lambda}=\frac{\zeta^{*}\left(\lambda_{1}-\lambda_{2}\right)}{\zeta^{*}\left(\lambda_{1}-\lambda_{2}+1\right)} \mathcal{I}_{\left(\lambda_{2}, \lambda_{1}, \lambda_{3}\right)}, \\
& M\left(\left(\begin{array}{ll}
1 & \\
1 & \\
& 1
\end{array}\right), \mathcal{I}_{\lambda}\right) \mathcal{I}_{\lambda}=\frac{\zeta^{*}\left(\lambda_{2}-\lambda_{3}\right)}{\zeta^{*}\left(\lambda_{2}-\lambda_{3}+1\right)} \frac{\zeta^{*}\left(\lambda_{1}-\lambda_{3}\right)}{\zeta^{*}\left(\lambda_{1}-\lambda_{3}+1\right)} \mathcal{I}_{\left(\lambda_{3}, \lambda_{1}, \lambda_{2}\right)} .
\end{aligned}
$$


The value of $E^{M_{\alpha}}$ at a point

$$
g=\left(\begin{array}{ccc}
1 & x_{2} & x_{3} \\
& 1 & x_{1} \\
& & 1
\end{array}\right)\left(\begin{array}{ccc}
y_{1} y_{2} & & \\
& y_{1} & \\
& & 1
\end{array}\right) k \in \operatorname{GL}(3, \mathbb{R})
$$

may be given in terms of the Eisenstein series

$$
e(\tau, s)=\sum_{\substack{(c, d)=1 \\ c>0}} \frac{y^{s}}{|c \tau+d|^{2 s}}
$$

for $\tau=x+i y$ in the upper half-plane. Specifically, if we let $\tau_{1}=x_{1}+i y_{1}$, then we find that

$$
E^{M_{\alpha}}\left(\mathcal{I}_{\lambda}, \mathcal{I}_{\lambda} \otimes 1\right)(g)=\left(y_{2} \sqrt{y_{1}}\right)^{\lambda_{1}+1} e\left(\tau_{1},\left(\lambda_{2}-\lambda_{3}+1\right) / 2\right) .
$$

It follows that the value of $E_{P_{\alpha}}\left(\mathcal{I}_{\lambda}\right)$ is given by

$$
\begin{aligned}
\left(y_{2} \sqrt{y_{1}}\right)^{\lambda_{1}+1} e\left(\tau_{1},\left(\lambda_{2}-\lambda_{3}+1\right) / 2\right) \\
\quad+\left(y_{2} \sqrt{y_{1}}\right)^{\lambda_{2}+1} \frac{\zeta^{*}\left(\lambda_{1}-\lambda_{2}\right)}{\zeta^{*}\left(\lambda_{1}-\lambda_{2}+1\right)} e\left(\tau_{1},\left(\lambda_{1}-\lambda_{3}+1\right) / 2\right) \\
\quad+\left(y_{2} \sqrt{y_{1}}\right)^{\lambda_{3}+1} \frac{\zeta^{*}\left(\lambda_{2}-\lambda_{3}\right)}{\zeta^{*}\left(\lambda_{2}-\lambda_{3}+1\right)} \frac{\zeta^{*}\left(\lambda_{1}-\lambda_{3}\right)}{\zeta^{*}\left(\lambda_{1}-\lambda_{3}+1\right)} e\left(\tau_{1},\left(\lambda_{1}-\lambda_{2}+1\right) / 2\right) .
\end{aligned}
$$

If we multiply $E\left(\mathcal{I}_{\lambda}, \mathcal{I}_{\lambda} \otimes 1\right)(g)$ by

$$
\zeta^{*}\left(\lambda_{1}-\lambda_{2}+1\right) \zeta^{*}\left(\lambda_{2}-\lambda_{3}+1\right) \zeta^{*}\left(\lambda_{1}-\lambda_{3}+1\right),
$$

then the resulting function is essentially the function $G_{\nu_{1}, \nu_{2}}$ appearing in Bump [B]. It is invariant under the six permutations of $\lambda_{1}, \lambda_{2}$, and $\lambda_{3}$. Its poles are along the lines $\lambda_{i}-\lambda_{j}=1$, and are permuted transitively by its functional equations. Let us therefore restrict our attention to the plane $\lambda_{2}-\lambda_{3}=1$.

Now, it is clear that of the three terms that make up $E_{P_{\alpha}}$, the first and third will be singular along this hyperplane, while the second will not. It is thus clear that

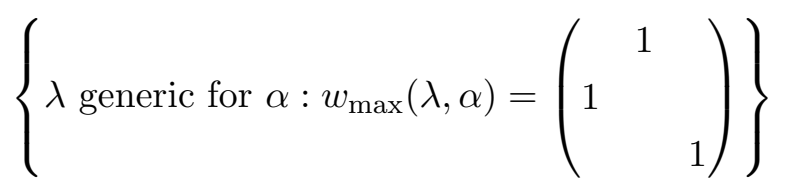

is the union of $\Omega_{1}:=\left\{\lambda \in H: \lambda_{2}>\lambda_{1}>\lambda_{3}\right\}$ and $\Omega_{2}:=\left\{\lambda \in H: \lambda_{1}<\lambda_{3}\right.$, $\left.\lambda_{3} \neq \lambda_{1}+1\right\}$. The function $e(\tau, s)$ does not vanish identically in $s$ for any $\tau$, and its residue is never zero. Thus, we may use any $p, k$. We fix $\lambda^{0} \in \Omega_{1} \cup \Omega_{2}$ such that $e\left(\tau_{1},\left(\lambda_{1}^{0}-\lambda_{3}^{0}+1\right) / 2\right) \neq 0$ and $\lambda^{1}$ in the hyperplane $\lambda_{2}-\lambda_{3}=0$, and define $\Lambda$ by $\Lambda(\sigma)=(1+\sigma) \lambda^{0}-\sigma \lambda^{1}$. There is no real loss of generality, since an arbitrary $\Lambda$ may be obtained from this one by a simple scaling. But this 
ensures that $\Lambda(\sigma)_{2}-\Lambda(\sigma)_{3}=\sigma+1$, so that the residue of $\zeta^{*}\left(\Lambda(\sigma)_{2}-\Lambda(\sigma)_{3}\right)$ at zero is 1 , while that of $e\left(\tau,\left(\Lambda(\sigma)_{2}-\Lambda(\sigma)_{3}+1\right) / 2\right)$ is

$$
\frac{1}{\zeta^{*}(2)}=\frac{6}{\pi} \text {. }
$$

If $\lambda^{0} \in \Omega_{1}$, then $w_{\mathrm{ms}}=I$. In this case, the asymptotic formula reads

$$
\beta \sim \frac{6 \zeta^{*}\left(\lambda_{1}^{0}-\lambda_{2}^{0}+1\right)}{\pi \zeta^{*}\left(\lambda_{1}^{0}-\lambda_{2}^{0}\right) e\left(\tau_{1},\left(\lambda_{1}^{0}-\lambda_{3}^{0}+1\right) / 2\right)\left(y_{2} \sqrt{y_{1}}\right)^{\lambda_{2}^{0}-\lambda_{1}^{0}}} .
$$

On the other hand, if $\lambda^{0} \in \Omega_{\alpha}^{2}$, then

$$
w_{\mathrm{ms}}=\left(\begin{array}{ccc} 
& & 1 \\
1 & & \\
& 1
\end{array}\right),
$$

and the asymptotic formula is

$$
\beta \sim \frac{6 \zeta^{*}\left(\lambda_{1}^{0}-\lambda_{2}^{0}+1\right) \zeta^{*}\left(\lambda_{1}^{0}-\lambda_{3}^{0}\right) e\left(\tau_{1},\left(\lambda_{1}^{0}-\lambda_{2}^{0}+1\right) / 2\right)}{\pi \zeta^{*}\left(\lambda_{1}^{0}-\lambda_{2}^{0}\right) \zeta^{*}\left(\lambda_{1}^{0}-\lambda_{3}^{0}+1\right) e\left(\tau_{1},\left(\lambda_{1}^{0}-\lambda_{3}^{0}+1\right) / 2\right)\left(y_{2} \sqrt{y_{1}}\right)} .
$$

\section{References}

[B-G1] P. T. Bateman and E. Grosswald, On Epstein's zeta function, Acta Arith. 9 (1964), 365-373.

[B-G2] -, -, Imaginary quadratic fields with unique factorization, Illinois J. Math. 6 (1962), 187-192.

[B] D. Bump, Automorphic Forms on GL(3, R), Lecture Notes in Math. 1083, Springer, Berlin, 1984.

[C-S1] S. Chowla and A. Selberg, On Epstein's zeta function, I, Proc. Nat. Acad. Sci. U.S.A. 35 (1949), 371-374.

[C-S2] -, - On Epstein's zeta-function, J. Reine Angew. Math. 227 (1967), 86-110.

[D-H] H. Davenport and H. Heilbronn, On the zeros of certain Dirichlet series, I, II, J. London Math. Soc. 11 (1936), 181-185, 307-312.

[G] D. Goldfeld, An asymptotic formula relating the Siegel zero and the class number of quadratic fields, Ann. Scuola Norm. Sup. Pisa Cl. Sci. 2 (1975), 611-615.

[G-S] D. Goldfeld and A. Schinzel, On Siegel's zero, ibid., 571-583.

[He] D. Hejhal, On a result of G. Pólya concerning the Riemann $\xi$-function, J. Analyse Math. 55 (1990), 59-95.

[Ho] J. Hoffstein, Real zeros of Eisenstein series, Math. Z. 181 (1982), 179-190.

[H-L] J. Hoffstein and P. Lockhart, Coefficients of Maass forms and the Siegel zero, Ann. of Math. 140 (1994), 161-181.

[J-S1] H. Jacquet and J. A. Shalika, On Euler products and the classification of automorphic representations, I, Amer. J. Math. 103 (1981), 499-558.

[J-S2] -, 一, On Euler products and the classification of automorphic forms, II, ibid., $777-815$.

[L-S] J. Lagarias and M. Suzuki, The Riemann hypothesis for certain integrals of Eisenstein series, J. Number Theory 118 (2006), 98-122. 
[L] R. Langlands, Euler Products, Yale Univ. Press, New Haven, CT, 1971.

[M-W] C. Møglin and J.-L. Waldspurger, Spectral Decomposition and Eisenstein Series, Cambridge Univ. Press, Cambridge, 1995.

[R-W] D. Ramakrishnan and S. Wang, On the exceptional zeros of Rankin-Selberg Lfunctions, Compos. Math. 135 (2003), 211-244.

[S1] H. M. Stark, On the zeros of Epstein's zeta function, Mathematika 14 (1967), $47-55$.

[S2] - A complete determination of the complex quadratic fields of class-number one, Michigan Math. J. 14 (1967), 1-27.

[T] A. Terras, The minima of quadratic forms and the behavior of Epstein and Dedekind zeta functions, J. Number Theory 12 (1980), 258-272.

[W] S. Wang, On the symmetric powers of cusp forms on GL(2) of icosahedral type, Int. Math. Res. Not. 2003, no. 44, 2373-2390.

Mathematics Department

Penn State University

University Park

State College, PA 16802, U.S.A.

E-mail: hundley@math.psu.edu

Received on 27.10.2005

and in revised form on 28.10.2006 